\title{
Pulsed Inductive Discharge as New Method for Gas Lasers Pumping
}

\section{Razhev AM ${ }^{1,2}$, Churkin DS ${ }^{1,2}$ and Kargapol'tsev ES ${ }^{1}$}

${ }^{1}$ Institute of Laser Physics SB RAS, Lavrentyeva ave. 13/3, Novosibirsk, 630090, Russia

${ }^{2}$ Novosibirsk State University, Pirogova st. 2, Novosibirsk, 630090, Russia

\begin{abstract}
Results of experimental investigations into the possibility of a pulsed inductive cylindrical discharge as a new method of pumping gas lasers operating at different transitions of atoms and molecules with different mechanisms of formation of inversion population are presented. The excitation systems of a pulsed inductive cylindrical discharge (pulsed inductively coupled plasma) in the gases are developed and experimentally investigated. For the first time five pulsed inductive lasers on the different transitions of atoms and molecules are created. Characteristic feature of the emission of pulsed inductive lasers is ring-shaped laser beam with low divergence and pulse-to-pulse instability is within $1 \%$.
\end{abstract}

Keywords: Pulsed inductive discharge; Gas lasers pumping; Pulsed inductively coupled plasma

\section{Introduction}

The RF induction excitation of continuous-wave lasing was reported in [1-3]. Continuous-wave lasing on transitions in atomic argon ions in the green spectral range under excitation by a longitudinal inductive $\mathrm{RF}$ discharge was obtained in [1,2]. Lasing on vibrational-rotational transitions in $\mathrm{CO}_{2}$ molecules in a wavelength range of $10.6 \mu \mathrm{m}$ in an expanding nitrogen flow heated by an inductive discharge after the addition of cold $\mathrm{CO}_{2}$ to it was reported in [3].

In this work, a method for exciting gas laser active media by a pulsed inductive cylindrical discharge is proposed and experimentally implemented in order to obtain lasing on electron transitions in atoms and molecules and vibrational-rotational transitions in molecules. It is important that the pulse repetition rate has to be several hertz or higher; i.e., all processes of discharge formation, creation of population inversion, amplification, absorption, and quenching must occur during each pulse irrespective of the past history of the preceding pulse. In contrast to conventional pulsed longitudinal and transverse electric discharges, a pulsed inductive cylindrical discharge is formed due to the magnetic field induction produced by the pumping system without any electrodes in the active medium. An appropriate choice of the tube material may ensure the purity of the active medium and considerable endurance of lasers. The formation of such a discharge is not accompanied by the appearance of cathode spots on the surface of the electrodes, which are responsible for the instability and contraction of the discharge, deterioration of the homogeneity of the discharge, contamination of the gas mixture, quenching of lasing, and limitation of the pulse repetition rate. The application of the pulsed inductive discharge for excitation is a promising method for pumping not only gas lasers, but also metal vapor and solid state lasers. In addition, this method can be used to produce the plasma for obtaining radiation (including induced radiation) in any spectral range, especially that extending from $100 \mathrm{~nm}$ to $\mathrm{THz}$, which is of considerable interest for microelectronics, photolithography and biomedicine.

\section{Apparatus}

In our measurements, the spontaneous emission spectra of the inductive discharge in gases and the lasing spectrum were recorded with a Ocean Optics HR 2000 spectrometer, S-150 Solar LS spectrometer with a resolution of $0.66 \mathrm{~nm}$ in the spectral range from 200 to 1100 $\mathrm{nm}$ and a SpectraPro-500 Acton Research Corp. spectrograph with a resolution of $0.025 \mathrm{~nm}$ in the spectral range from 180 to $700 \mathrm{~nm}$ with different photodiodes and photomultipliers. The output laser energy was measured with a PE50-BB Ophir pyroelectric pulse energy meter (Ophir Optronics Ltd). The temporal parameters of electric pulses were recorded with high-voltage P6015A probes and a 200-MHz TDS-2024 Tektronix oscilloscope. The accuracy of measurements was $5 \%$. The temporal parameters of optical pulses were recorded with a PhEC-22 and PhC-15 coaxial photocells with a temporal resolution of $10^{-10} \mathrm{~s}$ and infrared photodetector. The spatial distribution of the laser radiation intensity over the tube cross section and the light beam profile were analyzed by using a WinCamD-UCM digital video camera (Data Ray Inc).

\section{Experimental Setup}

In our experiments we used two excitation systems. The first one is shown in Figure 1. The excitation system operated in the following

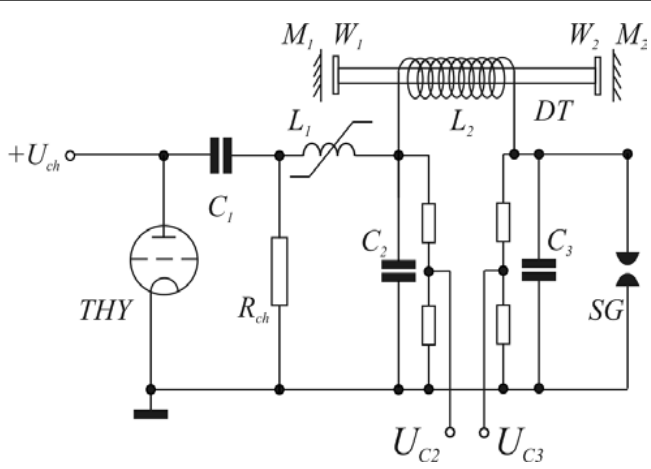

Figure 1: Electric circuit of the excitation system of pulsed inductive lasers. $T H Y$ - thyratron $T P I 1-10 k / 50, C_{1}=80 \mathrm{nF}, \boldsymbol{C}_{2}=8 \mathrm{nF}, \boldsymbol{C}_{3}=18 \mathrm{nF}, \boldsymbol{L}_{1}$ - chokingcoil, $L_{2}$ - inductor, $D T$ - discharge tube, $S G$ - self-triggered gas-filled spark gap.

*Corresponding author: Dmitry Churkin, Institute of Laser Physics SB RAS Lavrentyeva ave. 13/3, Novosibirsk, 630090, Russia, E-mail: churkin@laser.nsc.ru

Received August 05, 2013; Accepted September 19, 2013; Published September 23, 2013

Citation: Razhev AM, Churkin DS, Kargapol'tsev ES (2013) Pulsed Inductive Discharge as New Method for Gas Lasers Pumping. J Electr Electron Syst 2: 112 doi:10.4172/2332-0796.1000112

Copyright: (c) 2013 Razhev AM, et al. This is an open-access article distributed under the terms of the Creative Commons Attribution License, which permits unrestricted use, distribution, and reproduction in any medium, provided the original author and source are credited. 
way. The capacitor $C_{1}=80 \mathrm{nF}$ was charged from an ALE $152 \mathrm{~A}$ Lambda EMI pulsed power supply up to the voltage $20-27 \mathrm{kV}$ of the positive polarity. When the voltage across capacitor $C_{1}$ achieved the maximum, a triggering pulse was fed to a high-voltage switch (TPI1-10k/50 thyratron). After the thyratron switched on, the capacitor $C_{1}$ began to discharge, a negative voltage appeared across charging choking-coil $L_{1}$, and energy was transferred to capacitors $C_{2}$ and $C_{3}$. Both capacitors were charged during the time 1.5-2.0 $\mu$ s up to the breakdown voltage of a spark gap. The capacitor $C_{3}$ and the spark gap represented a lowinductive circuit in which the capacitor $C_{3}$ began to discharge after the actuation of the spark gap slightly earlier than the capacitor $C_{2}$. During the discharge of the latter, a time-varying electric current passed through the inductor $L_{2}$ placed on Discharge Tube DT and creates varying magnetic field around it, which induces azimuthally electric current in gases, leading to breakdown and formation of a pulsed inductive discharge (pulsed inductively coupled plasma).

This excitation system provided comparatively efficient energy transfer from peaking capacitors $C_{2}$ and $C_{3}$ to the active medium [4]. However this system contained nonlinear element $L_{1}$ which reduced total scheme efficiency. Moreover additional self-triggered spark gap $S G$ has not allowed to achieve high pulse-to-pulse stability and to operate with high pulse repetition rate.

To avoid these problems a new excitation system was developed (Figure 2). This system was based on well known scheme of Blumleintype. This excitation scheme had a very simple design and provided high stable operating conditions. Using this systems, inductive lasers had high pulse-to-pulse stability: amplitude differences were not more than $0.5 \%$.

The ignition moment of the inductive discharge was determined by the appearance of spontaneous emission of gases in the discharge tube. Under pressures below 1 Torr, inductive discharge filled the discharge tube completely, uniformly and had high intensity. As the mixture pressure increased above several Torr the intensity of inductive discharge in the center of the tube rapidly decreased. The discharge started to assume cylindrical form and concentrated near the inner tube wall surface. According to our observations discharge thickness decreased as a function of pressure. It was found in [4-8] that the output energy of the inductive lasers was proportional to the discharge tube diameter, i.e. the lasing efficiency and output energy increased with increasing the tube diameter. Because of this, we used in our experiments a ceramic discharge tube with maximal inner diameter we had ( $42 \mathrm{~mm}$, external diameter was $50 \mathrm{~mm}$ ). The tube was sealed by means of plane-parallel windows of $\mathrm{MgF}_{2}$ or $\mathrm{KCl}$ oriented perpendicular to the tube axis (Figure 1). The optical resonator was

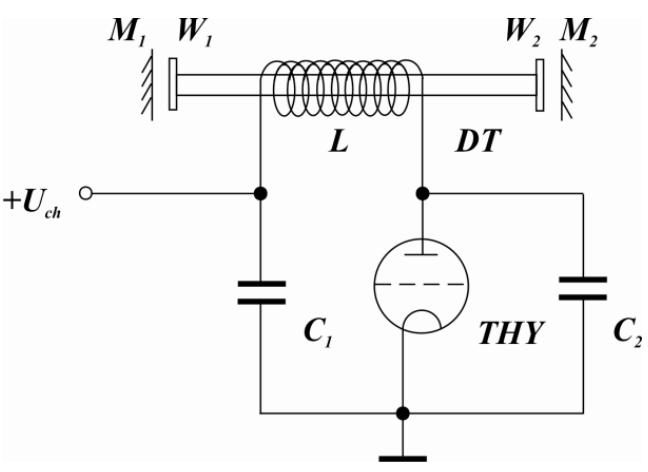

Figure 2: Electric circuit of the Blumlein-type excitation system. THY - thyratron $T P I 1-10 k / 50, C_{1}=18-30 \mathrm{nF}, C_{2}=30-55 \mathrm{nF}, \boldsymbol{L}$ - inductor, $\boldsymbol{D T}$ - discharge tube formed by external plane dielectric mirrors. The rear dielectric mirror had the reflectance $99 \%$ in the selected spectral region. The reflectance of the output mirror was optimized during experiments to obtain the maximum output energy. Mirrors with reflectance from $8 \%$ to $93 \%$ were used. The inductor $L_{2}$ consisted of separate sections representing solenoids made of a cable wire of cross section $1.5-6 \mathrm{~mm}^{2}$. The results presented in this paper were obtained by using the inductor containing 30 sections, each of them consisting of four coils. The solenoids were connected in parallel, and the total length $(\sim 68 \mathrm{~cm})$ of the inductor determined the length of the active medium of the gas laser. Gases or its mixtures were admitted from a gas system into the tube up to pressures 0.1-300 Torr. Gases flowed longitudinally during experiments.

\section{Results}

\section{Red laser on the electronic transitions of atomic fluorine}

In our first experiments to demonstrate the possibility of the creation of the pulsed inductive gas laser, we chose transitions in neutral fluorine atoms which works by the excitation of the pulsed cylindrical inductive discharge in a He: $\mathrm{F}_{2}\left(\mathrm{NF}_{3}\right)$ mixture, because the population inversion in these transitions is reached at comparatively low excitation levels in a wide pressure range [9-13]. In this case, a high gain is achieved, which ensures super luminescence regime in a low-Q resonator, and lasing takes place in the red spectral region and the transition from spontaneous emission to the lasing mode is easily detected.

In this work the developed laser on the electron excited transition of Fluorine (FI) atoms pumped by a pulsed inductive cylindrical discharge is described. Lasing at 8 wavelengths in range of $624-755 \mathrm{~nm}$ is obtained by exciting $\mathrm{He}-\mathrm{F}_{2}\left(\mathrm{NF}_{3}\right)$ gas mixtures in a pressure range from 20 to 300 Torr. The energy of laser radiation depended from the ratio of the mixture components and total pressure. The optimal composition for our pumping conditions was $\mathrm{He}_{2} \mathrm{~F}_{2}(80: 1)$ and total pressure 40-50 Torr. When $\mathrm{F}_{2}$ was replaced by $\mathrm{NF}_{3}$, lasing was also obtained, but with a lower intensity. For this reason, subsequent experiments were made on the He: $\mathrm{F}_{2}$ (80:1) mixtures. Maximum energy of FI laser in these experiments was achieved $2.6 \mathrm{~mJ}$ at pulse power $30 \mathrm{~kW}$ and durations $80 \mathrm{~ns}$. The laser beam divergence was $0.4 \mathrm{mrad}$.

\section{Ultraviolet laser on the self-limiting electronic transitions of the $\mathrm{N}_{2}$ molecules}

The purpose of this part of paper is to study the ability of the pulse periodic inductive cylindrical discharge to be a new method for pumping laser on self-limited electronic excited transitions of molecules and atoms (such as $\mathrm{N}_{2}, \mathrm{H}_{2}, \mathrm{He}$, metal vapor). The aim of this paper is to show that a pulsed inductive discharge is an efficient alternative tool for exciting UV nitrogen lasers, offering a number of advantages compared to transverse and longitudinal electric discharges.

Experiments with the pulsed inductive discharge in nitrogen showed that the pressure range in which the inductively coupled plasma can exist is quite narrow, from 0.1 to 10 Torr. Visual observations with the use of different optical filters showed that the inductive discharge was homogeneous in the entire pressure range. No sparks and streamers were observed in the inductive discharge in pure nitrogen and its mixtures. At pressures 0.2-3.0 Torr, the two most intense lines were observed at $337.1 \mathrm{~nm}$ and $357.7 \mathrm{~nm}\left(0-0\right.$ band $0-1$ band of $C^{3} \Pi$ $\rightarrow \boldsymbol{B}^{3} \Pi_{\text {transition respectively). After the mounting of dielectric mirrors }}$ and alignment of the optical resonator, we obtained UV lasing at these transitions of molecular nitrogen excited by the pulsed inductive discharge in the pressure range from 0.3 to 3 Torr. The maximum of the lasing efficiency was achieved at pressures $0.5-0.8$ Torr. The intensity of 
the $337.1 \mathrm{~nm}$ line exceeded that of the $357.7 \mathrm{~nm}$ line more than by two orders of magnitude. It is interesting that simultaneous lasing at these two lines in pure nitrogen at low pressures (1-2 Torr) was previously reported only in one paper [14]. The maximum output energy $6 \mathrm{~mJ}$ is achieved for resonator with front mirror with $\mathrm{R}_{2}=60 \%$. The laser pulse FWHM was $15 \pm 1 \mathrm{~ns}$. This corresponds to the laser pulse power of 500 $\mathrm{kW}$. As the reflectance of the output mirror was further increased, the output energy decreased. However, the laser pulse duration increased from $13 \pm 1 \mathrm{~ns}$ for $\mathrm{R}_{2}=16 \%$ to $18 \pm 1 \mathrm{~ns}$ for $\mathrm{R}_{2}=93 \%$. This is larger than in electric-discharge lasers, where the pulse duration does not exceed 5-10 ns $[15,16]$. The duration of UV laser pulses (at the base level) in the dense resonator exceeded $35 \mathrm{~ns}$. It is important to note that such a high pulsed power was never achieved before in a nitrogen laser at low pressures 1 Torr. This result demonstrates the specific features of the operation of the inductive nitrogen laser such as its emission spectrum containing many vibrational lines, which is obtained upon such excitation, and a long duration of pulses with a comparatively flat leading edge (about $7 \mathrm{~ns}$ ). According to our measurements, the pulseto-pulse instability of the lasing amplitude was $0.5 \%$. We performed experiments with the inductive nitrogen laser operating in the pulse periodic regime. The pulse repetition rate was varied from 1 to 30 $\mathrm{Hz}$. We found that the average output power increased linearly with increasing pulse repetition rate. For the repetition rate of $30 \mathrm{~Hz}$ and output power of the nitrogen laser of $6 \mathrm{~mJ}$, the average output power was $180 \mathrm{~mW}$. The output energy of a single pulse was independent of the pulse repetition rate because the active medium was cooled at the ceramic tube wall directly adjacent to the lasing region.

\section{Near infrared laser on the electronic transitions of the $\mathrm{H}_{2}$ molecules}

There are several works on lasing on the electronic transitions in $\mathrm{H}_{2}$ molecules in near IR spectra region $[17,18]$. Lasing was observed on 7 lines. Maximum peak power $1.5 \mathrm{~kW}$ was obtained. In our experiments lasing on the electronic transitions of $\mathrm{H}_{2}$ molecules in near IR spectra region with excitation by pulsed inductive discharge is achieved. The generation is observed on 4 lines. The wavelength $\lambda_{1}=0.835 \mu \mathrm{m}$ (band $(2,1)$ rotational line $P(2), \lambda_{2}=0.89 \mu \mathrm{m}$ (band $(1,0)$ rotational line $P(2)$, $\lambda_{3}=1.116 \mu \mathrm{m}$ (band $(0,0)$ rotational line $P(4)$, the $\lambda_{4}=1.122 \mu \mathrm{m}$ (band $(0,0)$ rotational line $P(2)$ that corresponds to $2 \mathrm{~s} \sigma^{1} \Sigma^{+}{ }_{\mathrm{g}}(E) \rightarrow(2 \mathrm{ps})^{21} \Sigma^{+}{ }_{\mathrm{g}}(B)$ transition. Maximum of laser emission peak power was at two lines: 7 $\mathrm{kW}$ for wavelength $\lambda_{2}=0.89 \mu \mathrm{m}$ and $5 \mathrm{~kW}$ for wavelength $\lambda_{4}=1.122 \mu \mathrm{m}$, respectively. Peak power of the lines $\lambda_{1}=0.835 \mu \mathrm{m}$ and $\lambda_{3}=1.116 \mu \mathrm{m}$ was much weaker. Pulse duration was $18-20 \mathrm{~ns}$ (FWHM). Laser is working both on one wavelength and on two wavelengths simultaneously with competition between these transitions. The active medium was hydrogen at optimal pressure 0.5-0.8 Torr.

\section{Mid and far infrared lasers on the vibrational-rotational transitions of the $\mathrm{HF}$ and $\mathrm{CO}_{2}$ molecules}

The purpose of this part of paper is to study the ability of the pulsed inductive cylindrical discharge to be a new method for pumping laser media and for creating the population inversion at vibrationalrotational transitions of molecules (such as $\mathrm{CO}_{2}, \mathrm{HF}$ or $\mathrm{DF}$ ) in the ground electronic state.

In the experiments we studied the effect of gas mixture composition on the lasing power characteristics of the inductive plasma HF laser. As a fluorine donor one used $\mathrm{F}_{2}, \mathrm{NF}_{3}$ and $\mathrm{SF}_{6}$, as hydrogen donor- $\mathrm{H}_{2}$. The highest laser power has been obtained with $\mathrm{SF}_{6}$ as the fluorine donor. The proportion of the components has been $\mathrm{H}_{2}: \mathrm{SF}_{6}-1: 2$. For the mixture of $\mathrm{H}_{2}-\mathrm{NF}_{3}$ lasing has also obtained, but its parameters were not studied due to dusting of the resonator's windows with the products of discharge. For $\mathrm{H}_{2}-\mathrm{F}_{2}$ we have not been able to obtain the infrared lasing. Comparing with electric discharge HF lasers, the pulsed inductive HF laser reaches its maximum of generation energy when $\mathrm{Ne}$ has added as a buffer gas in the mixture, but not He. During the experiments we have obtained $10 \mathrm{~mJ}$ for $\mathrm{H}_{2}-\mathrm{SF}_{6}-\mathrm{Ne}$ mixture, which is $10 \%$ higher than lasing in $\mathrm{H}_{2}-\mathrm{SF}_{6}-\mathrm{He}$ mixture.

To create the inductive $\mathrm{CO}_{2}$ laser in our experiments we used $\mathrm{CO}_{2}: \mathrm{N}_{2}: \mathrm{He}$ gas mixture as an active laser medium. The main purpose of our experiments was to investigate the temporal, energy, and spatial characteristics of pulsed inductive $\mathrm{CO}_{2}$ laser for gas mixtures with a different ratio of components as a function of the total pressure and excitation parameters.

Under total pressures of $10-15$ Torr of the gas mixture $\mathrm{CO}_{2}: \mathrm{N}_{2}: \mathrm{He}$ - 1:4:12 lasing on vibrational-rotational transitions of $\mathrm{CO}_{2}$ molecules at a wavelength of $10.6 \mathrm{~lm}$ in a pulsed inductive discharge has been observed. The maximum generation energy $152 \mathrm{~mJ}$ is obtained in a ceramic tube with an inner diameter of $42 \mathrm{~mm}$ in the $\mathrm{CO}_{2}: \mathrm{N}_{2}: \mathrm{He}-$ 1:4:12 gas mixture at a pressure of 15 Torr. The maximum efficiency in these first experiments did not exceed $0.3 \%$. We expect that it is possible to increase the inductive $\mathrm{CO}_{2}$ laser efficiency by using pulsed $\mathrm{RF}$ inductive discharge. The development of such excitation system is one of the goals of our further investigations. The measured divergence of $\mathrm{CO}_{2}$ laser radiation is $8 \mathrm{mrad}$. The possibility of the inductive discharge $\mathrm{CO}_{2}$ laser operation in the pulse-periodic regime was analyzed in the experiments. The pulse repetition rate varied from 1 to $50 \mathrm{~Hz}$. It was revealed that the average radiation power increases linearly with the pulse repetition rate. Thus an average power of $3.0 \mathrm{~W}$ is obtained at a repetition rate of $50 \mathrm{~Hz}$ and generation energy of $60 \mathrm{~mJ}$.

\section{Characteristics of the inductive discharge laser emission}

Characteristic feature of the emission of pulsed inductive lasers is ring-shaped laser beam. The thickness of the ring depends on active gas medium and pumping conditions. The study of the beam profile showed that the laser radiation intensity at the external boundary of the ring is lowest (Figure 3 ). The lasing intensity increases towards the ring

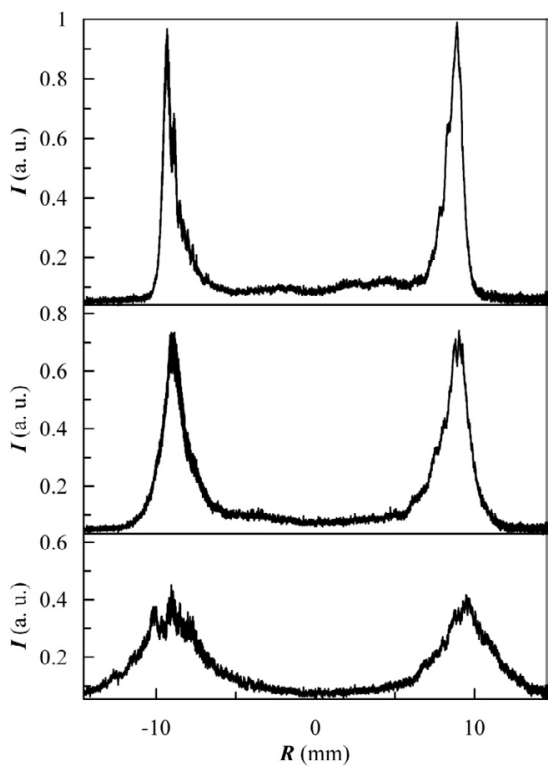

Figure 3: The profile of the laser beam of inductive discharge nitrogen lase at the distance $0.1 \mathrm{~m}, 4 \mathrm{~m}$ and $8 \mathrm{~m}$ from output window. Because of smal aperture of beam profiler, experiments were performed with discharge tube with inner diameter of $20 \mathrm{~mm}$. 
Citation: Razhev AM, Churkin DS, Kargapol'tsev ES (2013) Pulsed Inductive Discharge as New Method for Gas Lasers Pumping. J Electr Electron Syst 2: 112. doi:10.4172/2332-0796.1000112

centre and achieves the maximum at a distance of about $1 \mathrm{~mm}$ from the external boundary. Then, the radiation intensity decreases almost to zero at a distance of $4 \mathrm{~mm}$ from the boundary. This value can be treated as the ring width. The laser radiation divergence, which is determined by measuring the size of the laser radiation ring at different distances from the laser $0.1 \mathrm{~m}$ to $8 \mathrm{~m}$ and is $0,3 \div 8 \mathrm{mrad}$ in dependence of the laser wavelength. The circular structure of the beam cross section is a specific feature of pulsed inductive lasers with a cylindrical inductive discharge. Such beams in the case of a low radiation divergence offer certain advantages because they can be focused to produce the radiation intensity distribution similarly to Bessel beams.

\section{Conclusion}

A pulsed inductive cylindrical discharge as a new method of pumping gas lasers operating at different transitions of atoms and molecules with different mechanisms of formation of inversion population is proposed and experimentally realized. The excitation systems of a pulsed inductive cylindrical discharge (pulsed inductively coupled plasma) in the gases are developed and experimentally investigated. For the first time five pulsed inductive lasers on the different transitions of atoms and molecules were created. Characteristic feature of the emission of pulsed inductive lasers is ring-shaped laser beam with low divergence and pulse-to-pulse instability is within $0.5 \%$.

\section{References}

1. Bell WE (1965) Ring discharge excitation of gas ion lasers. Appl Phys Lett 7: 190-191.

2. Goldborough JP, Hodges EB, Bell WE (1966) RF induction excitation of CW visible laser transition in ionized gases. Appl Phys Lett 8: 137-139.

3. Kiselevskii LI, Skutov DK, Sokolov SA (1974) Primenenie visokochastotnogo indukzionnogo razrjada dla poluchenija lasernoi generazii $v$ neprerivnom regime. Zh Prikl Spektroskopiya 21: 951-955.
4. Razhev AM, Churkin DS, Zhupikov AA (2009) Study of the UV emission of an inductive nitrogen laser. Quantum Electronics 39: 901-905.

5. Razhev AM, Mkhitaryan VM, Churkin DS (2005) 703-to 731-nm FI laser excited by a transverse inductive discharge. JETP Letters 82: 259-262.

6. Razhev AM, Churkin DS, Zavyalov AS (2009) Pulsed inductive discharge molecular hydrogen laser. Vestnik NSU Seria Fizika 4: 12-19.

7. Razhev AM, Churkin DS (2009) Pulsed inductive discharge $\mathrm{CO}_{2}$ laser. Opt Commun 282: 1354-1357.

8. Razhev AM, Churkin DS (2007) Inductive ultraviolet nitrogen laser. JETP Lett 86: 420-423.

9. Hocker LO, Phi TB (1976) Pressure dependence of the atomic fluorine laser transition intensities. Appl Phys Lett 29: 493-494.

10. Loree TR, Sze RC (1977) The atomic fluorine laser: spectral pressure dependence. Opt Commun 21: 255-257.

11. Lisitsin VN, Razhev AM (1977) High-power, high-pressure laser based on red fluorine lines. Pisma v Zhurnal Tekhnischeskoi Fiziki 3: 862-864.

12. Lawler JE, Parker JW, Anderson LW, Fitzsimmons WA (1979) Experimenta investigation of the atomic fluorine laser. IEEE J Quantum Electron 15: 609 613

13. Zaeferani MS, Parvin P, Sadighi R (1996) Pressure dependence of the spectral lines of a high power, high pressure atomic fluorine laser pumped by a charge transfer from $\mathrm{He}_{2}^{+}$. Opt Laser Technol 28: 203-205

14. Kaslin VM, Petrash GG (1966) Rotational structure of ultraviolet generation of molecular nitrogen. JETP Letters 3: 88-92.

15. Shipman JD (1967) Traveling wave excitation of high power gas lasers. App Phys Lett 10: 3-4.

16. Wang CP (1976) Simple fast-discharge device for high-power pulsed lasers Rev Sci Instrum 47: 92-95.

17. Bazhulin PA, Knyazev IN, Petrash GG (1965) Stimulated radiation from hydrogen and deuterium molecules in the near infrared region. JETP 49: 16-23.

18. Bockasten K, Lundholm T, Andrade O (1966) Laser lines in atomic and molecular hydrogen. J Opt Soc Am 56: 1260-1261. 